\title{
Mikrostrukturelle Charakterisierung einer pulvermetallurgisch hergestellten mehrphasigen TiAl-Legierung
}

\author{
Michael Burtscher ${ }^{1}$, Fabian Pürstl ${ }^{1}$, Martin Schloffer², Svea Mayer ${ }^{1}$ und Helmut Clemens ${ }^{1}$ \\ 'Department Werkstoffwissenschaft, Montanuniversität Leoben, Leoben, Österreich \\ ${ }^{2} \mathrm{MTU}$ Aero Engines AG, München, Deutschland
}

Eingegangen 8. Februar 2019; angenommen 11. März 2019; online publiziert 27. März 2019

Zusammenfassung: Intermetallische Titanaluminide werden bei erhöhten Temperaturen als Turbinenschaufeln in Flugzeugtriebwerken, als Laufräder in Turboladern sowie als Ventile im Rennsport eingesetzt. Um neue Herstellungswege zu nutzen und endkonturnahe 3D gedruckte Teile produzieren zu können, müssen geeignete Pulver für additive Fertigungsprozesse verfügbar sein. Dazu wurden zwei vielversprechende Legierungsvarianten herangezogen und entsprechende Pulver hergestellt. Anschließend wurden die Pulver heißisostatisch verdichtet. Um die Qualität der Pulver sicherzustellen und mögliche mikrostrukturelle Inhomogenitäten des Materials auszuschließen, erfolgte eine Defektanalyse mittels Lichtmikroskopie in Kombination mit Rasterelektronenmikroskopie. Dazu wurden einzelne Defekte im Lichtmikroskop mittels Härteeindrücken markiert und deren lokale chemische Information über detaillierte Flächenscans im Rasterelektronenmikroskop ermittelt. Die Ergebnisse dieser Untersuchung können dazu verwendet werden, Fehlerquellen bei der Herstellung sensibler Titanaluminid-Pulver zu vermeiden und so die Qualität der erzeugten Bauteile zu steigern.

Schlüsselwörter: Intermetallische Legierungen, Titanaluminide, Pulvermetallurgie, Mikrostrukturelle Charakterisierung

Microstructural Characterisation of a Multi-Phase TiAIAlloy Produced by Powder Metallurgy

Abstract: Intermetallic $\gamma$-TiAl based titanium aluminides are used at elevated temperatures as turbine blades in aero engines as well as turbocharger wheels and valves in au-

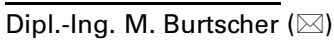

Department Werkstoffwissenschaft,

Montanuniversität Leoben,

Franz-Josef-Straße 18,

8700 Leoben, Österreich

michael.burtscher@unileoben.ac.at tomotive applications. In order to fulfil the aim for near net shaped and 3D printed parts, suitable powders have to be available. Therefore, powders of two different promising alloying systems were produced. These powders were HIPed to achieve a dense bulk material. To ensure quality and exclude any possible microstructural inhomogeneity of the bulk material, a defect analysis with a light optical microscope was performed in combination with a scanning electron microscope. For that, any microstructural inhomogeneity was marked with an indent in the surrounding matrix and afterwards energy-dispersive $x$-ray spectroscopy was used to achieve the local chemical information of these defects. The results of this analysis can be used to avoid the sources of defects within the production of sensitive TiAl powders and, therefore, increase the quality of produced components.

Keywords: Intermetallic alloys, Titanium aluminides, Powder metallurgy, Microstructural characterisation

\section{Einleitung}

Die gegenwärtige Entwicklung, komplexe Bauteile über die additive Fertigung herzustellen, um dadurch den Materialeinsatz zu minimieren und die Endbearbeitung der Teile zu reduzieren, geht auch auf Materialsysteme wie Titanaluminid (TiAl)-Legierungen über [1-4]. Die in dieser Arbeit verwendeten Legierungen bestehen zum größten Teil aus der intermetallisch geordneten $\gamma$-TiAl-Phase und weisen ein optimales Eigenschaftsprofil für Hochtemperaturanwendungen in der Luftfahrt sowie Automobilindustrie auf [5]. In diesem Bereich haben sie das Potential etablierte NiBasislegierungen zu substituieren, da sie in etwa die Hälfte der Dichte, gute Oxidations- sowie Kriecheigenschaften und eine hohe spezifische Festigkeit besitzen $[5,6]$. Vor allem die hohe spezifische Festigkeit ermöglicht ein schnelleres Ansprechverhalten von beschleunigten Teilen in Au- 
tomobilen, z.B. Turboladerlaufrädern und Ventilen, und bietet zudem durch Reduktion des Gewichts im Flugzeugbau ein Einsparungspotential von Treibhausgasen, wie $\mathrm{CO}_{2}$ und $\mathrm{NO}_{x}$ [7]. Um den strengen Emissionsvorschriften zu entsprechen und einen entscheidenden Kostenvorteil gegenüber den im Betrieb befindlichen Triebwerken zu erlangen, werden diese Werkstoffe bereits von den führenden Triebwerksherstellern in modernen Flugzeugturbinen eingesetzt. Bei der konventionellen Herstellung der TiAlTurbinenschaufeln wird ein mehrfach umgeschmolzenes Vormaterial über einen Umformschritt auf eine endkonturnahe Form gebracht [5]. Das Gussverfahren begrenzt seigerungsbedingt die maximale Größe der Bauteile. Durch eine Herstellung über die additive Formgebung kann diese Größenbarriere aufgrund der kleinen Schmelzpoolgröße im additiven Herstellprozess auf die Dimension des Bauraums der 3D Drucker erhöht und gleichzeitig eine größere Formenvielfalt erreicht werden. Bei dieser Herstellungsart kommt es jedoch aufgrund der Pulverqualität und der noch mangelnden Erfahrung hinsichtlich der Prozessparameter zur Ausbildung von unterschiedlichen Defekten [8-10]. Um den zyklischen Belastungen innerhalb der geplanten Lebensdauer eines Triebwerks standzuhalten, dürfen die Gefüge jedoch keinesfalls größere Defekte aufweisen, als dies bei konventionell erzeugten Bauteilen der Fall ist. Ziel dieser Studie ist es daher, die Pulver auf ihre Eignung für eine additive Fertigung zu untersuchen und auftretende Defekte zu identifizieren.

\section{Experimentelles}

Die zu untersuchenden TiAl-Pulver wurden über das VIGA(Vakuum-Induktionsschmelzen mit Inertgas-Verdüsung) Verfahren hergestellt. Dazu werden die Vorlegierungen sowie Legierungselemente in einem Vakuuminduktionsofen, dem sogenannten „Skull Melter”, unter Inertgas erhitzt. Dieser Vorgang erfolgt induktiv, sorgt im Schmelzbad für eine ausreichende Durchmischung und führt dadurch zu einer homogenen Schmelze. Anschließend wird diese über einen geheizten Kanal zum Zerstäuber geführt, wo sie mithilfe eines starken Inertgasstroms pulverisiert wird. Bei der Fertigung über einen additiven Prozess werden besonders homogene Pulver, welche von gleichbleibender Qualität sind, gebraucht. Daher wurde die verwendete Pulverfraktion von 45 bis $125 \mu \mathrm{m}$ im Querschliff sowie als solche im Rasterelektronenmikroskop (REM) vom Typ Evo50 der Firma Zeiss, Deutschland, mittels Rückstreuelektronen (RE) auf Poren, Form und Satelliten untersucht. Der Schliff wurde durch Vermengen von Pulverpartikel mit gemörserter PolyFast Einbettmasse von Struers, Dänemark, realisiert. Durch lockeres Aufbringen der Pulver auf ein Kupferband und anschließendes Bedampfen mit Gold konnten die Oberflächen der Partikel im REM unter Sekundärelektronenkontrast (SE) untersucht werden. Die Analyse der Größenverteilung erfolgte durch eine Partikelgrößenmessung mittels Laserbeugung mit dem Gerät HELOS der Firma Sympatec, Deutschland. Anschließend wurden die verschiedenen Pulver nach üblichem Stand der Technik bei einer Temperatur von $1200^{\circ} \mathrm{C}$ und $200 \mathrm{MPa}$ über eine Dauer von $4 \mathrm{~h}$ heißisostatisch gepresst (HIP) [5].

Die dadurch erhaltenen zylindrischen und dichten Proben wurden mit einem Trennschleifer vom Typ AbrasiMatic 300 der Firma Buehler, Deutschland, entsprechend Abb. 1 getrennt und anschließend metallographisch präpariert. Hierzu wurde die Stirnseite der Probe mit Schleifpapier mit einer Körnung von 500, 800, 1000, 2000 und 4000 nass geschliffen und mittels $3 \mu \mathrm{m}$ sowie $1 \mu \mathrm{m}$ Diamantsuspension poliert. Danach wurden die Proben mit einem Elektropoliergerät vom Typ LectroPol-5 und unter Verwendung des A3-Elektrolyt von Struers, Dänemark, sowie einer nachfolgenden Farbätzung nach [11] für großflächige lichtmikroskopische (LIMI) Aufnahmen präpariert. Anhand dieser konnte die Position der Defekte gefunden und nachfolgend durch drei Härteeindrücke (HV0.1) mit einem Mikrohärteprüfer Micro-Dumat 4000 der Firma Reichert-Jung, Deutschland, und einem Makrohärteeindruck (HV10) von einem Universalhärtemesser vom Typ M4C 025 G3M der Firma EMCO Test, Österreich, markiert werden (Abb. 1). Um die Defekte auch auf ihre Chemie hin zu untersuchen, musste die durch die vorhergehende Ätzung aufgebrachte Oxidschicht wieder entfernt werden. Diese konnte durch erneutes Polieren mit Diamantsuspension ( 3 und $1 \mu \mathrm{m}$ ) und nachfolgendem $30-40$ s Polieren mit OPS beseitigt werden. Durch die vorhergehende Markierung konnten die einzelnen Defekte im REM gefunden und mittels energiedispersiver Röntgenspektroskopie (EDX) flächenmäßig analysiert werden. Bei dem verwendeten EDX System handelt es sich um das X-Max ${ }^{N}$ Messsystem der Firma Oxford Instruments, England.

\section{Ergebnisse und Diskussion}

\subsection{Charakterisierung der Pulver}

Um die Eignung des VIGA Prozesses zur Herstellung von TiAl-Pulver zu verifizieren, wurden diese mittels REM und Laserbeugungsmessungen klassifiziert. In Abb. 2a und b sind SE Aufnahmen der Oberflächen und RE Aufnahmen der Schliffe der gesiebten Pulverfraktion dargestellt.

Die Pulverpartikel besitzen teilweise Satelliten und zeigen vereinzelt spratzige Anhaftungen. Beide Merkmale wirken sich negativ auf die Rieselfähigkeit aus und behindern so eine optimale Verteilung im Pulverbett [12]. Die Oberflächen weisen unterschiedlich große Domänen auf, deren Ursprung auf Dendritenbildung während der Erstarrung zurückgeführt werden kann [13]. Die Größe der Domänen ist unabhängig von der Pulvergröße und setzt sich innerhalb der Partikel fort. An den Domänengrenzen kommt es zu einer Anreicherung von Al während der Erstarrung [13]. In den Querschliffen konnten vereinzelt Inhomogenitäten festgestellt werden, welche mittels Pfeile markiert sind. Der Anteil an Partikel mit inneren Poren ist äußerst gering und wurde daher in dieser Arbeit vernachlässigt. Die beiden betrachteten Legierungen besitzen eine sehr ähnliche GröBenverteilung der Pulverpartikel und sind daher gut miteinander vergleichbar. Die Mediane der Normalverteilungen liegen mit $67 \mu \mathrm{m}$ (Leg. 1) und $69 \mu \mathrm{m}$ (Leg. 2) relativ mittig 
Abb. 1: Skizze über den Ablauf der Probenentnahme aus den geHIPten Rohlingen und Schema zur Markierung der zu analysierenden Defekte
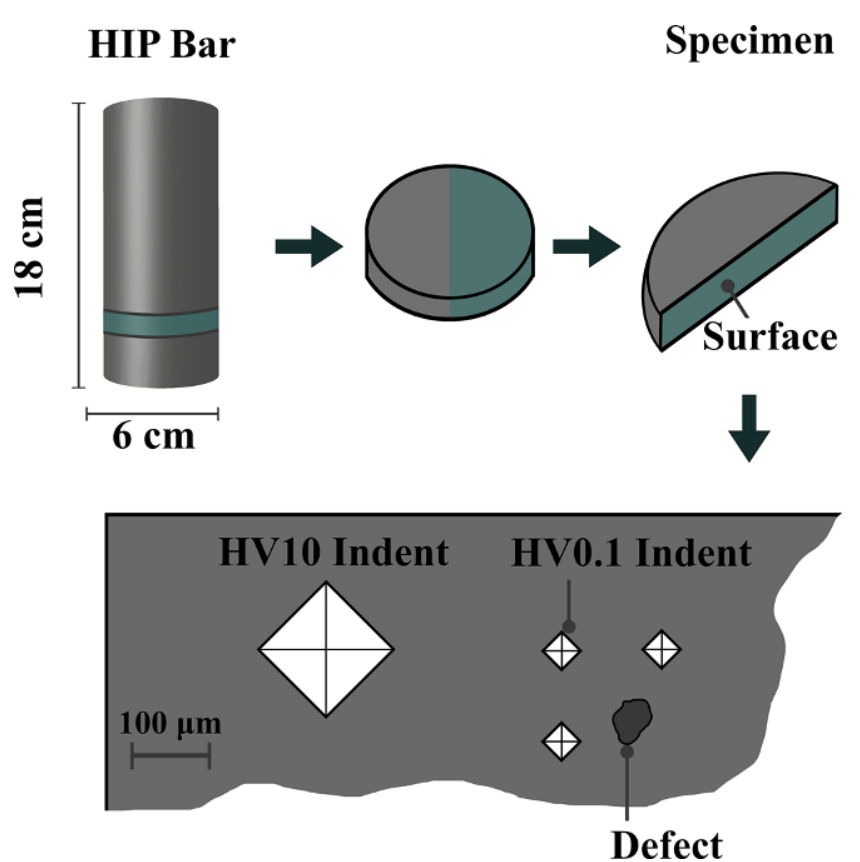

Abb. 2: Darstellung der Pulverpartikel im SE Kontrast und deren Querschliffe als RE Aufnahmen der Legierung 1 in a und Legierung 2 in b. Dabei konnten Domänenstrukturen, Satelliten, sogenannte "Shells" sowie vereinzelt innere Defekte, welche mit Pfeilen gekennzeichnet sind, an den Pulvern gefunden werden
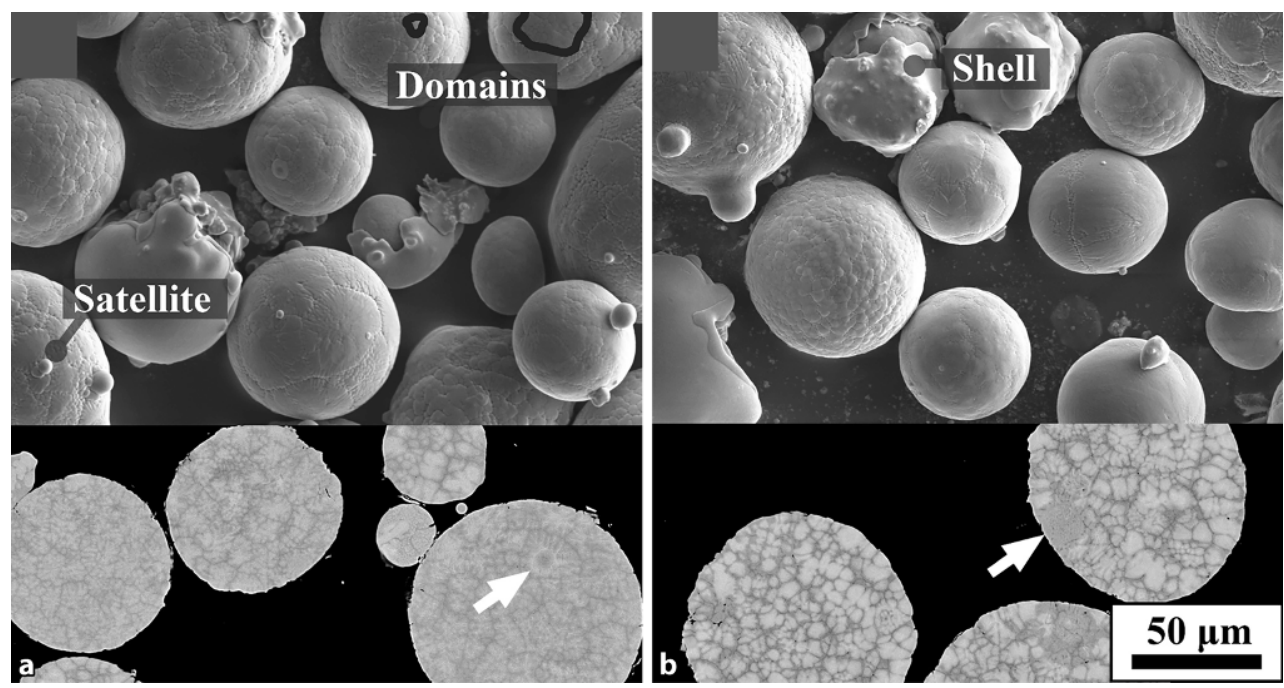

in der gewünschten Siebfraktion (Abb. 2). Daher entspricht auch der größte Anteil der Pulverpartikel mit annähernd $80 \%$ der gewünschten Fraktion von $45-125 \mu \mathrm{m}$.

\subsection{Defektanalyse der Mikrostruktur}

Ein additiver Fertigungsschritt wurde in dieser Untersuchung bewusst nicht durchgeführt, um die Einflüsse aus dem Herstellungsprozess der Pulver genauer analysieren und Artefakte aus dem Druckprozess ausschließen zu können. Um dennoch ein dichtes Material für weitere Analysen zu erhalten, wurden die Pulver durch einen HIP Vorgang zu Rohlingen verdichtet (Abb. 1). In der Mikrostruktur der geHIPten Rohlinge kann die für Pulver typische Domänenstruktur nicht mehr festgestellt werden. Dies lässt vermuten, dass die Al Mikroseigerungen durch die Wärme- behandlung gleichmäßig verteilt wurden. Allerdings treten in beiden Legierungen Defekte auf, welche durch Zulegieren von hochschmelzenden Legierungselementen ( $\mathrm{Nb}$, Mo) entstanden sein könnten [10, 12]. Diese Legierungselemente sind ausschlaggebend für die Anwendbarkeit des untersuchten Legierungstyps und sind dabei besonders für die gute Umformbarkeit bei hohen Temperaturen und auch für den Erhalt einer homogenen Mikrostruktur durch die Erstarrung über die $\beta$ Phase verantwortlich [5]. Aufgrund der Differenz der Schmelzpunkte zu Al und Ti werden die Legierungselemente als Vorlegierungen - in Kombination mit anderen Elementen - hinzugegeben. Können diese Vorlegierungen nicht gänzlich aufgeschmolzen werden, kommt es zu charakteristischen Defekten, wie es in [10] bereits für $\mathrm{Nb}$ Anreicherungen gezeigt wurde. In Abb. 3 ist in (a) ein Mo und in (b) ein W reicher Defekt dargestellt. Wegen ihrer ähnlichen Wirkung auf das Legierungssys- 
Abb. 3: Darstellung eines an Mo und W angereicherten Defekts aus den untersuchten Legierungen 1 und 2 . Bei der in den REM Bildern (RE-Kontrast) hell erscheinenden Phase handelt es sich um die durch diese Elemente stabilisierte $\beta_{0}$ Phase. Die EDX Flächenscans lassen durch ortsaufgelöste Information der Al und W Gehalte Aussagen über die Elementverteilung, Größe und Matrixinteraktion in den Defekten zu
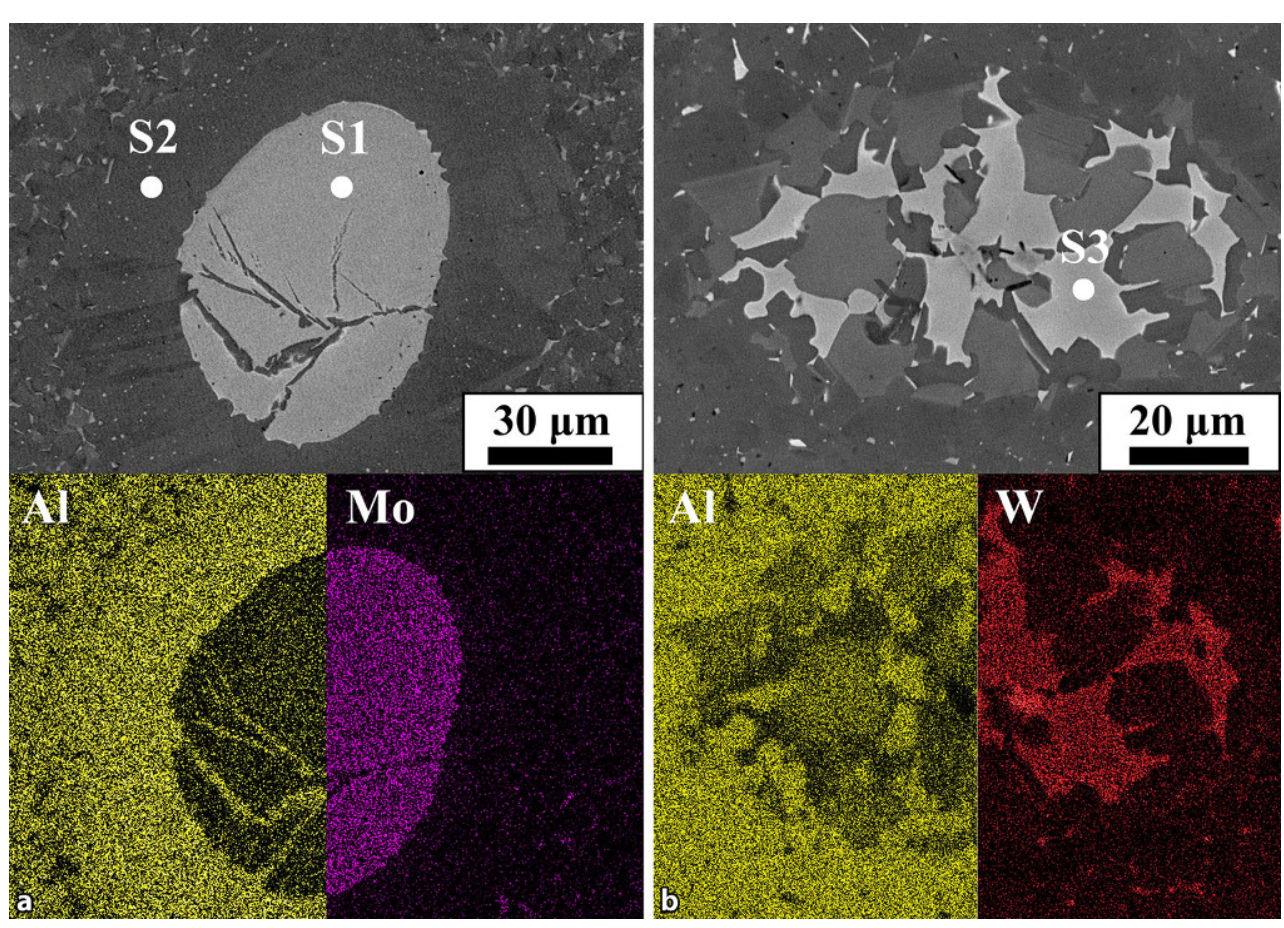

tem stabilisieren beide Elemente die bei Raumtemperatur spröde und geordnete $\beta_{\circ}$ Phase [14]. Über die Darstellung von Al im EDX Flächenscan können durch eine Kontraständerung die Grenzen der Einflusszone des Defekts ermittelt werden, wodurch die Kontraständerung zur Bestimmung der Defektgröße herangezogen werden kann. Die Größe der Defekte in Legierung 1 reicht von 75 bis $133 \mu \mathrm{m}$ und beträgt durchschnittlich $95 \mu \mathrm{m}$, während in Legierung $2 \mathrm{~W}$ haltige Defekte von 85 bis $124 \mu \mathrm{m}$ auftreten und durchschnittlich $105 \mu \mathrm{m}$ groß sind. Innerhalb der Mo-reichen Defekte kann in Abb. 3a eine $\gamma$ Phase gefunden werden, welche sich während des HIP Prozesses bildet [15]. Die chemische Zusammensetzung der Defekte wurde an ausgewählten Punkten quantitativ bestimmt und beträgt an Position S1 etwa 10 at.\% Mo, was dem 10-fachen der nominellen Zusammensetzung dieser Legierung entspricht. An Position S2 nimmt der Mo Gehalt auf etwa 1,7 at.\% ab und normalisiert sich außerhalb des $\gamma$-Saums mit 1,3 at.\% auf den Wert, welcher konstant über die Probe gemessen wurde. In Abb. 3b ist ein ähnlicher Defekt dargestellt, welcher jedoch an $\mathrm{W}$ angereichert ist. In der durch $\mathrm{W}$ stabilisierten $\beta_{0}$ Phase konnte an Position S3 ein Anteil von 3,6 at.\% gemessen werden, was dem ca. 3-fachen der angestrebten Zusammensetzung entspricht. Etwa $25 \mu \mathrm{m}$ neben dem Defekt fällt der Wert auf 1,1 at.\% W ab, was wiederum mit dem gewünschten Legierungsgehalt übereinstimmt. Bei näherer Betrachtung enthält der Defekt aus Legierung 2 auch globulare $\alpha_{2}$ und $\gamma$ Körner, aber keinen definierten $\gamma$ Saum und auch keinen scharfen Übergang zwischen dem Defekt und der umliegenden Matrix. Dies kann durch den niedrigeren W Gehalt im Vergleich zu dem in Legierung 1 vorkommenden Mo-Defekt erklärt werden. In beiden Fällen konnte das elementar eingebrachte Refraktärmetall nicht durch die nachfolgende Homogenisierung des HIP Prozes- ses verteilt werden, da die beiden Elemente eine geringere Neigung zur Diffusion besitzen als das zuvor erwähnte Al [12].

\section{Zusammenfassung}

In diesem Beitrag wurden zwei unterschiedliche TiAl-Legierungspulver auf ihre Eignung zur Herstellung von 3D gedruckten Teilen in Hinblick auf das Auftreten von Defekten untersucht. Dabei konnten im Querschliff der Ausgangspulver mittels REM vereinzelt Inhomogenitäten gefunden werden. Durch HIPen wurden dichte und quasi unbeeinflusste Proben für großflächige LIMI Untersuchungen aus den gesiebten Pulvern hergestellt. Darin konnten Inhomogenitäten mit einer durchschnittlichen Größe von ca. $100 \mu \mathrm{m}$ und einer maximalen Größe von bis zu $133 \mu \mathrm{m}$ gefunden werden. Um ausgewählte Defekte im REM wieder zu finden, konnten diese mittels Härteeindruck dauerhaft markiert werden. Danach wurden die markierten Inhomogenitäten mittels EDX Flächenscan analysiert und entsprechend der vorkommenden Legierungselemente analysiert und ausgewertet. Dazu stellte sich besonders der Al-Gehalt als ein guter Indikator heraus, da dieser sensibel auf Phasenänderungen und daher auch auf auftretende Defekte reagiert. Darüber hinaus konnten die Verteilung der Elemente, die Größe der Inhomogenität und auch deren Anbindung zur Matrix untersucht werden. Defekte mit einem Durchmesser von $100 \mu \mathrm{m}$ sind für hochbelastete Bauteile als kritisch anzusehen, da sie zurzeit nicht zerstörungsfrei im Bauteil nachgewiesen werden können. Durch einen additiven Prozessschritt kommt es zu einem nochmaligen Aufschmelzen der Pulver und damit auch der eingebrachten W- und Mo-haltigen Partikel, was zu einer Beeinflussung 
der Größe der Defekte führen kann. Wie sehr diese dadurch ausgebildeten Reaktionszonen die Eigenschaften des Bulkwerkstoffes beeinflussen, ist Gegenstand einer weiterführenden Untersuchung. Aus den Ergebnissen dieser Studie ist ableitbar, dass für additiv gefertigte Bauteile, die aus Pulvern der Fraktion 45-125 $\mu \mathrm{m}$ gefertigt werden, neue zerstörungsfreie Prüftechniken und Methoden entwickelt werden müssen, um im Pulver sowie im Bauteil Kontaminationsprodukte und Defektgrößen detektieren bzw. bewerten zu können. Basierend auf dieser Arbeit konnten die Auslöser für zwei Defektarten in den Mikrostrukturen der beiden Legierungen gefunden werden. Dadurch ergab sich die Möglichkeit bei der Herstellung der Pulver Verbesserungen in der Prozessführung zur Verhinderung dieser Defekte durchzuführen.

Funding. Open access funding provided by Montanuniversität Leoben.

Open Access Dieser Artikel wird unter der Creative Commons Namensnennung 4.0 International Lizenz (http://creativecommons.org/licenses/ by/4.0/deed.de) veröffentlicht, welche die Nutzung, Vervielfältigung, Bearbeitung, Verbreitung und Wiedergabe in jeglichem Medium und Format erlaubt, sofern Sie den/die ursprünglichen Autor(en) und die Quelle ordnungsgemäß nennen, einen Linkzur Creative Commons Lizenz beifügen und angeben, ob Änderungen vorgenommen wurden.

\section{Literatur}

1. Todai, M.; Nakano, T.; Liu, T.; Yasuda, H. Y.; Hagihara, K.; Cho, K.; Ueda, M.; Takeyama, M.: Effect of building direction on the microstructure and tensile properties of Ti-48Al-2 $\mathrm{Cr}-2 \mathrm{Nb}$ alloy additively manufactured by electron beam melting, Additive Manufacturing, 13 (2017), pp 61-70

2. Biamino, S.; Penna, A.; Ackelid, U.; Sabbadini, S.; Tassa, O.; Fino, P.; Pavese, M.; Gennaro, P.; Badini, C.: Electron beam melting of $\mathrm{Ti}-48 \mathrm{Al}-2 \mathrm{Cr}-2 \mathrm{Nb}$ alloy: Microstructure and mechanical properties investigation, Intermetallics, 19 (2011), no 6, pp 776-781

3. Jabbar, H.; Monchoux, J.-P.; Thomas, M.; Pyczak, F.; Couret, A.: Improvement of the creep properties of TiAl alloys densified by Spark Plasma Sintering, Intermetallics, 46 (2014), pp 1-3

4. Doubenskaia, M.; Domashenkov, A.; Smurov, I.; Petrovskiy, P.: Study of Selective Laser Melting of intermetallic TiAl powder using integral analysis, International Journal of Machine Tools and Manufacture, 129 (2018), pp 1-14
5. Mayer, S.; Erdely, P.; Fischer, F. D.; Holec, D.; Kastenhuber, M.; Klein, T.; Clemens, H.: Intermetallic $\beta$-Solidifying $\gamma$-TiAl Based Alloys-From Fundamental Research to Application, Adv. Eng. Mater., 19 (2017), no 4, pp 1-27

6. Clemens, H.; Mayer, S.: Design, Processing, Microstructure, Properties, and Applications of Advanced Intermetallic TiAl Alloys, Adv. Eng. Mater., 15 (2013), no 4, pp 191-215

7. Masiol, M.; Harrison, R. M.: Aircraft engine exhaust emissions and other airport-related contributions to ambient air pollution: A review, Atmos. Environ., 95 (2014), pp 409-455

8. Baudana, G.; Biamino, S.; Ugues, D.; Lombardi, M.; Fino, P.; Pavese, M.; Badini, C.: Titanium aluminides for aerospace and automotive applications processed by Electron Beam Melting: Contribution of Politecnico di Torino, Metal Powder Report, 71 (2016), no 3, pp 193-199

9. Seifi, M.; Salem, A. A.; Satko, D. P.; Ackelid, U.; Semiatin, S. L.; Lewandowski, J. J.: Effects of HIP on microstructural heterogeneity defect distribution and mechanical properties of additively manufactured EBM Ti-48AI-2Cr-2Nb, Journal of Alloys and Compounds, 729 (2017), pp 1118-1135

10. Gasper, A.N.D.; Catchpole-Smith, S.; Clare, A. T.: In-situ synthesis of titanium aluminides by direct metal deposition, Journal of Materials Processing Technology, 239 (2017), pp 230-239.

11. Schloffer, M.; Schmoelzer, T.; Mayer, S.; .Schwaighofer, E.; Hawranek, G.; Schimansky, F.-P.; Pyczak, F.; Clemens, H.: The characterisation of a powder metallurgically manufactured $\mathrm{TNM}^{\mathrm{TM}}$ titanium aluminide alloy using complimentary quantitative methods, Pract. Metallogr., 48 (2011), no 11, pp 595-604

12. Simonelli, M.; Aboulkhair, N. T.; Cohen, P.; Murray, J. W.; Clare, A. T.; Tuck, C.; Hague, R. J. M.: A comparison of Ti-6Al-4V in-situ alloying in Selective Laser Melting using simply-mixed and satellited powder blend feedstocks, Materials Characterization, 143 (2018) pp. 118-126

13. Kastenhuber, M.; Klein, T.; Rashkova, B.; Weißensteiner, I.; Clemens, H.; Mayer, S.: Phase transformations in a $\beta$-solidifying $\gamma$-TiAl based alloy during rapid solidification, Intermetallics, 91 (2017), pp 100-109

14. Klein, T.; Clemens, H.; Mayer, S.: Advancement of Compositional and Microstructural Design of Intermetallic $\gamma$-TiAl Based Alloys Determined by Atom Probe Tomography, Materials, 9 (2016) pp. 755

15. Cheng, T. T.; Loretto, M. H.: The decomposition of the beta phase in $\mathrm{Ti}-44 \mathrm{Al}-8 \mathrm{Nb}$ and $\mathrm{Ti}-44 \mathrm{Al}-4 \mathrm{Nb}-4 \mathrm{Zr}-0.2 \mathrm{Si}$ alloys, Acta Mater., 46 (1998), no 13, pp 4801-4819

Hinweis des Verlags. Der Verlag bleibt in Hinblick auf geografische Zuordnungen und Gebietsbezeichnungen in veröffentlichten Karten und Institutsadressen neutral. 